\title{
Prognostic Impact of the Number of Peritumoral Alveolar Macrophages in Patients With Stage I Lung Adenocarcinoma
}

\section{Osamu Noritake}

National Cancer Center-Hospital East: Kokuritsu Gan Center Higashi Byoin

\section{Keiju Aokage}

National Cancer Center-Hospital East: Kokuritsu Gan Center Higashi Byoin

\section{Ayako Suzuki}

Tokyo Daigaku - Kashiwa Campus

\section{Kenta Tane}

National Cancer Center-Hospital East: Kokuritsu Gan Center Higashi Byoin

\section{Tomohiro Miyoshi}

National Cancer Center-Hospital East: Kokuritsu Gan Center Higashi Byoin

\section{Joji Samejima}

National Cancer Center-Hospital East: Kokuritsu Gan Center Higashi Byoin

\section{Toyohumi Yoshikawa}

Nagoya University Graduate School of Medicine Faculty of Medicine: Nagoya Daigaku Daigakuin Igakukei Kenkyuka Igakubu

\section{Tokiko Nakai}

National Cancer Center-Hospital East: Kokuritsu Gan Center Higashi Byoin

Masahiro Tsuboi

National Cancer Center-Hospital East: Kokuritsu Gan Center Higashi Byoin

Genichiro Ishii ( $\nabla$ gishii@east.ncc.go.jp )

National Cancer Center Hospital East https://orcid.org/0000-0003-4158-0286

\section{Research Article}

Keywords: Alveolar macrophage, Peritumoral, Lung adenocarcinoma, The Cancer Genome Atlas

Posted Date: September 7th, 2021

DOl: https://doi.org/10.21203/rs.3.rs-844311/v1

License: (c) (1) This work is licensed under a Creative Commons Attribution 4.0 International License. Read Full License 


\section{Abstract}

Purpose:

Intratumoral macrophages are reportedly involved in tumor progression in non-small cell lung cancer; however, little is known about the prognostic impact and function of alveolar macrophages (AMs). This study aims to investigate the prognostic impact of the number of peritumoral AMs in patients with stage I lung adenocarcinoma.

\section{Methods:}

We investigated 514 patients with pathological stage I lung adenocarcinoma who underwent complete resection with lobectomy or pneumonectomy. The number of peritumoral AMs were counted, and patients were classified into two groups based on the number of peritumoral AMs. Using the Cancer Genome Atlas (TCGA) database of stage I lung adenocarcinoma, we compared gene expression profiles of high and low peritumoral AM contents.

Results:

The median number of peritumoral AMs per alveolar space was 15.5. Patients with a high peritumoral AM content had significantly shorter disease-free survival and overall survival than patients with a low peritumoral AM content (both $p<0.01$ ). In the multivariate analyses, a higher number of peritumoral AMs was an independent prognostic factor $(p=0.02)$. The analysis of TCGA database revealed that patients with a high peritumoral AM content had shorter disease-free survival than those with a low peritumoral AM content $(p=0.04)$. Gene expression analysis of TCGA stage I lung adenocarcinoma revealed enrichment of biological processes, such as chemotaxis and epithelial proliferation, in patients with a high peritumoral AM content.

\section{Conclusion:}

The number of peritumoral AMs had a strong impact on disease-free survival in patients with stage I lung adenocarcinoma.

\section{Introduction}

Lung cancer is the leading cause of cancer-related deaths worldwide (Siegal et al. 2020). Non-small lung cancer (NSCLC) accounts for approximately $80 \%$ of all lung malignancies (Lewis et al. 2014), and adenocarcinomas are the most common type of NSCLC. Early-stage NSCLC patients are generally treated with complete surgical resection of the tumor. However, even after the complete resection of stage I NSCLC patients, the 5-year survival rate was $81-92 \%$ (Rami-Porta et al. 2014). Therefore, it is important to identify risk factors for recurrences that are different from conventional viewpoints, such as invasive tumor size, lymphovascular invasion, and pleural invasion. 
In addition to tumor cells, tumor tissues are composed of lymphocytes, monocytes, macrophages, and fibroblasts, establishing a complex tumor microenvironment (TME) (Comit et al. 2020; Kalluri. 2016; Shinghal et al. 2019). Not only tumor cells but also non-tumor cells influence tumor progression (Ishii et al. 2016; Hwang et al. 2020). Macrophages are one of the cells that make up TME, and are known to be closely involved in tumorigenesis and progression of lung cancer (Lewis et al. 2006; Bingle et al. 2002; Mantovani et al. 2017; Conway et al. 2016). Intratumoral CD204-positive tumor associated macrophages (TAMs) have been reported to be associated with tumor aggressiveness in lung adenocarcinoma, and squamous cell carcinoma (Ohtaki et al. 2010; Hirayama et al. 2012).

Additionally, some studies have reported that the peritumoral microenvironment influences tumor progression. In pancreatic cancer, the expression of secreted protein acidic and rich in cysteine (SPARC) by peritumoral fibroblasts indicates a poorer prognosis for patients (Infante et al. 2007). Moreover, hyaluronan in peritumoral stroma is associated with cancer cell spreading in breast cancer (Auvinen et al. 2000). Peritumoral macrophages promote tissue remodeling and proangiogenic pathways in hepatocellular carcinoma, by inducing Th17-mediated inflammation in peritumoral stroma (Kuang et al. 2010). For small cell lung cancer, Iriki reported that the macrophages that accumulated near the peripheral areas of tumor nests are important for tumor progression via STAT3 activation, suggesting that peritumoral macrophages may be deeply involved in tumor progression (Iriki et al. 2017).

Alveolar macrophages (AMs) reside in the alveolar space, and playing a crucial role in lung homeostasis (Lee et al. 2015; Allard et al. 2018). Additionally, AMs are major cells that constitute the peritumoral microenvironment. In the mouse model, AMs perform self-renewal throughout their life cycle (Guilliams et al. 2013, unlike stromal macrophages that are recruited from the circulation (Pollard et al. 2004; Sica et al. 2012). In breast cancer, AMs secrete transforming growth factor-beta in a premetastatic niche, promoting lung metastases (Sharma et al. 2015). Moreover, AMs mainly advance lung metastasis by generating leukotriene B4 in hepatocellular carcinoma (Nosaka et al. 2018). Speth et al found SOCS3 secreted by AMs inhibits tumor progression in lung cancer (Speth et al. 2019).

Based on these findings, we hypothesized that peritumoral AMs are associated with the prognosis of lung cancer. Thus, this study aims to investigate the prognostic impact of the number of peritumoral AMs in patients with stage I lung adenocarcinoma.

\section{Materials And Methods}

\section{Patients}

Patients with pathological stage I adenocarcinoma who underwent lobectomy or pneumonectomy with systemic lymph node dissection at the National Cancer Center East between January 2011 and December 2015 were enrolled in this study (Supplementary Fig. 1). We excluded patients who received preoperative therapy and those with adenocarcinoma in situ, undetectable tumors in surgical specimens, and synchronous lung cancer. Finally, 514 patients were enrolled in this study. The study was approved by the 
institutional review board of the National Cancer Center Hospital East (IRB approval number 2020 - 239). The clinical characteristics of patients were retrospectively retrieved from the Thoracic Surgical Database of the National Cancer Center Hospital East.

\section{Pathologic evaluation}

All surgical specimens were fixed in $10 \%$ formalin, and embedded in paraffin. All tumors were cut at 5-mm intervals, and $4 \mu \mathrm{m}$ thick-sections were stained using the hematoxylin and eosin (HE) method. The Verhoeff-van-Gieson (VVG) method was used to visualize elastic fibers to identify lymphovascular and pleural invasion. Histological diagnosis was based on the fourth edition of the World Health Organization series, and the disease stages were categorized according to the guidelines of the 8th edition of the TNM Classification of Malignant Tumors.

\section{Calculation of the number of alveolar macrophages}

We selected the largest cross-sectional slide for each patient to calculate the number of AMs (Figs. 1A-D). If the tumor was on multiple slides in the largest cross-section, we counted all these slides. The HE slides were scanned and captured using a digital slide scanner (Aperio VERSA SL200; Leica, Biosystems, Nußloch, Germany) and were reviewed by two pathologists (O.N and G.I) who were blinded to the clinicopathologic information of each slide. The peritumoral alveolar space was defined as the air space outside the tumor within three alveoli, and the number of macrophages per alveolar space was counted. AMs were distinguished from spread through air spaces (STAS) of tumor cells using the following methods. Macrophages in smokers typically have cytoplasm containing faint brown pigment and black carbon granules, whereas in nonsmokers the pigment is lacking and the cytoplasm is sometimes foamy. Nuclei are small, uniform, and regular, without atypia. Nuclear folds are frequent, and nucleoli are inconspicuous or absent. Contrastingly, STAS generally lack cytoplasmic pigment or foamy cytoplasm. They often grow in cohesive clusters and nuclei are atypical with hyperchromasia and frequent nucleoli. The number of peritumoral AMs was determined as the average of the top five AMs in each patient.

We investigated the correlation between the number of peritumoral AMs in HE-stained and peritumoral CD68-positive AMs. Forty patients were examined for CD68 expression in the peritumoral alveolar space (Supplementary Figs. 2A, B). The distribution of peritumoral AMs on HE slides and CD68-positive macrophages in each patient strongly correlated $\left(p<0.01, r^{2}=0.91\right)$ (Fig. 1E). Therefore, we assessed that HE slides were efficient for counting the number of peritumoral AMs, and later the number of peritumoral AMs was evaluated using HE slides only.

\section{Gene expression signature analysis on the Cancer Genome Atlas data sets}

We analyzed the difference in gene signatures between the number of AMs in lung adenocarcinoma, using the Cancer Genome Atlas-Lung Adenocarcinoma (TCGA- LUAD) datasets. All analyses were performed using R software (version 4.0.2) (https://www.r-project.org/; The R Foundation for Statistical Computing, Vienna, Austria). We downloaded the data of 155 patients with stage I lung adenocarcinoma 
in June 2020 from the Genomic Data Common (GDC) Data Portal (https://portal.gdc.cancer.gov) using the R/Bioconductor package TCGA biolinks. Using representative digital slides of each patient that can be viewed on the site, we counted and calculated peritumoral AMs and divided them into two groups in the manner described above. Twenty-five patients were excluded because of difficulty in evaluating peritumoral AMs. Differentially expressed genes between the high and low AM groups were identified using the DESeq2 package (version 1.28.1) [Love MI et al]. Genes were extracted using adjusted p-values (padj) of $<0.05$ and $\mid \log 2$ fold changes $(\log 2 \mathrm{FC}$ ) | of $>1$. We performed hierarchical clustering of genes that differed in expression between the high and low AM groups ( $|\log 2 \mathrm{FC}|>1)$, and heatmap analysis was performed using gplots (version 3.0.4) [Warnes GR et al.] and genefilter (version 1.70.0) [Gentleman R et al.] packages. Additionally, the differentially expressed genes were assessed for enrichment analysis using Metascape (http://metascape.org/gp/index.html\#/main/step1) [Zhou Y et al]. The Metascape pathway enrichment analysis used terms across different ontology sources, such as Gene Ontology (GO).

\section{Statistical analysis}

Associations between the clinicopathologic variables and the number of peritumoral AMs were analyzed using Fisher's exact test (for categorical variables) and the Mann-Whitney $U$ test (for continuous variables). The number of peritumoral AMs in each predominant subtype group was compared using the Mann-Whitney $U$ test. Pearson correlation coefficient was used to evaluate the correlation between the number of peritumoral AMs on HE slides and the number of CD68-positive peritumoral AMs. To evaluate prognosis, receiver operating characteristic curves of the number of peritumoral AMs were used. Overall survival (OS) was defined as the interval between the date of surgery and the date of death from any cause or the last follow-up. Disease-free survival (DFS) was defined as the time between the date of surgery and the date of recurrence detection, death from any cause, or the last follow-up. Survival curves were estimated using the Kaplan-Meier method, and differences in OS and DFS were compared by using the log-rank test. Hazard ratios (HRs) were estimated using the Cox proportional hazards model. The date of data cut-off was May 2019 at our institution. These data were analyzed using EZR version 1.51, a graphical user interface for $\mathrm{R}$ program.

\section{Results}

\section{Correlation between clinicopathological factors and the number of peritumoral AMs}

The median number of peritumoral AMs was 15.5 per alveolar space, and we divided patients into two groups based on this value. Patients with a higher number of peritumoral AMs were men, with smoking history; high-grade pathologic T stage; larger pathologic total and invasive tumor sizes; the presence of pleural invasion, lymph vessel invasion and vascular invasion; and a higher proportion of invasive predominant subtypes (Table 1). 
Table 1

Clinicopathologic characteristics of patients with high-vs low-peritumoral AMs

\begin{tabular}{|c|c|c|c|c|}
\hline & Overall & peritumoral & peritumoral & \\
\hline & Cohort & AMs $<15.5$ & $\mathrm{AMs} \geq 15.5$ & \\
\hline Variables & $(n=514)$ & $(n=257)$ & $(n=257)$ & $p$ \\
\hline \multicolumn{5}{|l|}{ Age(y) } \\
\hline median & $69(33-93)$ & $68(35-87)$ & $69(33-93)$ & \\
\hline$<65$ & 152 & 75 & 77 & 0.92 \\
\hline$\geq 65$ & 362 & 182 & 180 & \\
\hline \multicolumn{5}{|l|}{ Sex } \\
\hline female & 250 & 149 & 101 & $<0.01$ \\
\hline male & 264 & 108 & 156 & \\
\hline \multicolumn{5}{|l|}{ Smoking history } \\
\hline never & 232 & 136 & 96 & $<0.01$ \\
\hline ever/current & 282 & 121 & 161 & \\
\hline \multicolumn{5}{|l|}{ Pathologic T stage } \\
\hline $1 \mathrm{mi} / 1 \mathrm{a} / 1 \mathrm{~b} / 1 \mathrm{c}$ & $51 / 122 / 153 / 70$ & $39 / 82 / 82 / 23$ & $12 / 40 / 71 / 47$ & $<0.01$ \\
\hline $2 a$ & 118 & 31 & 87 & \\
\hline Total tumor size(mm) & & & & $<0.01$ \\
\hline median(range) & $24(7-75)$ & $22(7-62)$ & $25(8-75)$ & \\
\hline Invasive tumor size(mm) & & & & $<0.01$ \\
\hline median(range) & $14(1.5-40)$ & $11(1.5-40)$ & $18(2-40)$ & \\
\hline Pleural invasion & & & & $<0.01$ \\
\hline absent & 411 & 230 & 181 & \\
\hline present & 103 & 27 & 76 & \\
\hline Lymphatic permeation & & & & $<0.01$ \\
\hline absent & 480 & 250 & 230 & \\
\hline present & 34 & 7 & 27 & \\
\hline Vascular invasion & & & & $<0.01$ \\
\hline
\end{tabular}




\begin{tabular}{|lllll|}
\hline & Overall & peritumoral & peritumoral & \\
\hline absent & 411 & 238 & 173 & $<0.01$ \\
\hline present & 103 & 19 & 84 & \\
Predominant subtype & & & & \\
Lepidic & 260 & 180 & 80 & \\
Papillary & 115 & 42 & 73 & 0.36 \\
Micropapillary & 1 & 1 & 0 & \\
Acinar & 81 & 27 & 54 & \\
Solid & 57 & 7 & 50 & \\
EGFR mutation $(\mathrm{n}=244)$ & & & 75 & \\
\hline absent & 120 & 45 & 54 & \\
\hline present & 124 & 54 & & \\
\hline
\end{tabular}

\section{Correlation between the numbers of peritumoral AMs and the predominant subtype}

The relationships between the number of peritumoral AMs and predominant subtypes are presented in Fig. 2, wherein the micropapillary-predominant subtype was excluded because of one case. The median number of peritumoral AMs for the predominant subtype was 9.6 in lepidic, 20.4 in papillary, 20.2 in acinar, 27.2 in solid subtypes. The number of peritumoral AMs in the predominantly solid subtype was significantly higher than that in the acinar subtype $(p<0.01)$, papillary subtype $(p<0.01)$, and lepidic subtype $(p<0.01)$. There were no significant differences between the number of peritumoral AMs in the predominantly acinar and papillary subtypes $(p=0.78)$, and the number of peritumoral AMs in these two subtypes was significantly higher than that in the lepidic subtype (both $p<0.01$ ).

\section{Survival by the number of peritumoral AMs}

The median follow-up time of patients was 61.8 months. Patients with a high peritumoral AM content showed significantly shorter OS ( $p<0.01,5$-year OS rate: $95 \%$ vs. $83.4 \%)$ (Fig. $3 A$ ) and DFS $(p<0.01,5$ year DFS rate: $91.3 \%$ vs. $73.5 \%$ ) (Fig. 3B) than patients with a low peritumoral AM content in stage I adenocarcinoma. In stage IA, patients with a high AM content showed significantly shorter OS $(p=0.02$, 5 -year OS rate: $95.3 \%$ vs. $88.7 \%$ ) (Fig. $3 C$ ) and DFS ( $p<0.01$, 5-year DFS rate: $93.2 \%$ vs. $81.4 \%$ ). In stage IB, patients with a high peritumoral AM content showed significantly shorter OS ( $p=0.03,5$-year OS rate: $92.4 \%$ vs. $72.8 \%$ ) (Fig. 3D) and DFS ( $p=0.03$, 5-year OS rate: $77.2 \%$ vs. $57.8 \%$ ) (Fig. 3F). 
In the univariate analysis, a larger invasive tumor size; the presence of pleural invasion, lymphatic permeation, presence of vascular invasion; and a higher number of peritumoral AMs were significantly associated with shorter DFS (Table 2). 
Table 2

Univariate and multivariate analysis for disease-free survival based on the number of peritumoral AMs

\begin{tabular}{|c|c|c|c|c|c|c|}
\hline \multirow[t]{2}{*}{ Variable } & \multicolumn{3}{|c|}{ Univariate } & \multicolumn{3}{|c|}{ Multivariate } \\
\hline & HR & $95 \% \mathrm{Cl}$ & $p$ & HR & $95 \% \mathrm{Cl}$ & $p$ \\
\hline \multicolumn{7}{|l|}{ Age(y) } \\
\hline$<65$ & ref. & & & & & \\
\hline$\geq 65$ & 1.31 & $0.83-2.08$ & 0.24 & & & \\
\hline \multicolumn{7}{|l|}{ Sex } \\
\hline Female & ref. & & & & & \\
\hline Male & 1.32 & $0.88-1.98$ & 0.18 & & & \\
\hline \multicolumn{7}{|c|}{ Smoking history } \\
\hline Never & ref. & & & & & \\
\hline Ever/Current & 1.29 & $0.85-1.94$ & 0.23 & & & \\
\hline \multicolumn{7}{|c|}{ Invasive tumor size(cm) } \\
\hline$(+1.0 \mathrm{~cm})$ & 2.25 & $1.83-2.78$ & $<0.01$ & 1.60 & $1.23-2.07$ & $<0.01$ \\
\hline \multicolumn{7}{|c|}{ Pleural invasion } \\
\hline absent & ref. & & & ref. & & \\
\hline present & 3.12 & $2.11-4.81$ & $<0.01$ & 1.14 & $0.69-1.86$ & 0.62 \\
\hline \multicolumn{7}{|c|}{ Lymph vessel invasion } \\
\hline absent & ref. & & & ref. & & \\
\hline present & 3.02 & $1.77-5.18$ & $<0.01$ & 1.37 & $0.79-2.40$ & 0.26 \\
\hline \multicolumn{7}{|c|}{ Vascular invasion } \\
\hline absent & ref. & & & ref. & & \\
\hline present & 5.07 & $3.39-7.58$ & $<0.01$ & 2.55 & $1.56-4.24$ & $<0.01$ \\
\hline \multicolumn{7}{|c|}{ Number of peritumoral AMs } \\
\hline low $(<15.5)$ & ref. & & & ref. & & \\
\hline high ( $\geq 15.5)$ & 3.44 & $1.93-4.80$ & $<0.01$ & 1.85 & $1.11-3.09$ & 0.02 \\
\hline
\end{tabular}


In the multivariate analysis, a higher number of peritumoral AMs was an independent prognostic factor for DFS (HR 1.85, 95\% confidence interval (Cl): 1.11-3.09, $p=0.02$ ), in addition to invasive tumor size and vascular invasion.

\section{Evaluation of the survival impact of the number of peritumoral AMs}

Patients were stratified into two groups according to the different cut-off values of the number of peritumoral AMs. Multivariate analyses showed that although the cut-off value was 10 , there was no significant difference between patients with high and low peritumoral AMs. Both the cut-off values of 20 and 30 were independent factors for DFS (HR 2.01, 95\% confidence interval: 1.28-3.15, $p<0.01$; HR 3.27, $95 \% \mathrm{Cl}: 2.10-5.08, p<0.01$, respectively), and the hazard ratio gradually increased as the cut-off value increased (Table 3 ). This result suggested the possibility that the higher the number of peritumoral AMs, the more likely the tumor would recur.

Table 3

Summary of the results of multivariate analyses for disease-free survival about the number of peritumoral AMs

\begin{tabular}{|lllll|}
\hline Number of peritumoral AMs & $\mathbf{n}$ & HR & $95 \%$ Cl & $p$ \\
\hline$>10$ vs. $\leqq 10$ & & & & \\
\hline$>10$ & 182 & ref. & & \\
$\leqq 10$ & 332 & 1.32 & $0.75-2.34$ & 0.33 \\
\hline$>15.5$ vs. $\leqq 15.5$ (Median) & & & & \\
\hline$>15.5$ & 257 & ref. & & \\
$\leqq 15.5$ & 257 & 1.85 & $1.11-3.09$ & 0.02 \\
\hline$>20$ vs. $\leqq 20$ & & & & \\
\hline$>20$ & 321 & ref. & & \\
\hline 520 & 193 & 2.01 & $1.28-3.15$ & $<0.01$ \\
\hline$>30$ vs. $\leqq 30$ & & & & \\
\hline$>30$ & & & & \\
\hline$\leqq 30$ & 413 & ref. & & \\
\hline
\end{tabular}

\section{Survival and gene ontology analysis by the number of peritumoral AMs in TCGA database}


Of the 130 patients, the median number of peritumoral AMs that we calculated on TCGA digital slides was 11.3, and we divided them into two groups using this value. Patients with a higher number of peritumoral AMs showed significantly shorter DFS ( $p=0.04)$ (Fig. 4A), whereas there was no significant difference in OS (data not shown).

We extracted 212 differentially expressed genes (DEGs) between patients with high and low peritumoral AMs. A heatmap of 212 DEGs is presented in Fig. 4B. The Metascape pathway enrichment analysis was performed, which identified the top 20 biological processes in the DEGs, including chemotaxis (GO: 0006935) and epithelial proliferation (GO: 0050673) (Fig. 4C).

\section{Discussion}

In our study, clinicopathological factors showed that patients with a high peritumoral AM content had more aggressive tumors, such as with larger pathologic invasive tumor size; the presence of pleural invasion, lymphatic permeation, and a higher proportion of more invasive predominant subtype, than patients with a low peritumoral AM content. The multivariate analysis revealed that the number of peritumoral AMs was an independent prognostic factor. These results suggest that the number of peritumoral AMs is a strong factor for poor prognosis in patients with stage I lung adenocarcinoma. To the best of our knowledge, this is the first report showing that the number of AMs is associated with the prognosis of stage I lung adenocarcinoma.

In the analysis using the TCGA dataset, patients with a high peritumoral AM content showed shorter DFS than those with a low peritumoral AM content, indicating that the results using our hospital cohort are reproducible. The significant difference in some GO pathways, such as chemotaxis and epithelial proliferation, might be one of the reasons why the prognosis was poorer in the group with a high peritumoral AM content.

Previous reports have described the relationship between the tumor and the microenvironment around the tumor. In some types of cancer, peritumoral macrophages facilitate the progression in tumor cells (Kuang et al. 2010; Iriki et al. 2017). These reports indicate that the microenvironment around the tumor, not only within the tumor, is involved in tumor progression. Alternatively, Sharma et al. reported that AMs provide an environment where lung metastases are likely to occur by suppressing antitumor $T$ cell responses in breast cancer (Sharma et al. 2015). Future studies are needed to examine the gene expression profiles of AMs around tumors.

We suggest two possible reasons why the number of peritumoral AMs differs depending on the patient: (1) Cancer cells themselves mobilize the peritumoral AMs, and the ability to attract AMs varies depending on the characteristics of cancer cells in each case. (2) AMs that are originally present in alveoli before tumorigenesis and the characteristics of AMs in each case may influence the development of adenocarcinoma. To verify our theory, we made the following considerations: We defined nonadjacent alveolar space as the air space $2 \mathrm{~mm}$ away from the tumor edge on the largest cross-sectional slide, to avoid overlapping with the peritumoral alveolar space, and counted macrophages per alveolar space 
(Supplementary Figs. 3A, B). There was a strong correlation between peritumoral and nonadjacent AMs $\left(r^{2}=0.723\right.$; Supplementary Fig. 3C). The median number of nonadjacent AMs was 8.6 per alveolar space. In the multivariate analysis, there were no significant differences between patients with a high and low non-adjacent AM content with the cut-off values of 8.6 and 10 ( $p=0.54$ and 0.50 , respectively) (Supplementary Tables 1, 2). For the cut-off value of 20, patients with a high nonadjacent AM content showed poorer DFS than those with a low nonadjacent AM content (HR 1.57, 95\% Cl: 1.21-2.04, $p<0.01$ ) (Supplementary Table 2). This result may indicate that highly malignant tumors are likely to occur in cases with many AMs where the tumor develops. However, we cannot rule out the possibility that AMs $2 \mathrm{~mm}$ apart may also be mobilized by cancer cells. Further studies are needed to elucidate why the number of peritumoral AMs varies for each case, further studies will be needed.

There are some limitations to our study. First, this was a retrospective study in a single institution. Second, we did not evaluate the number of AMs on all slides with tumors. Third, the number of AMs is affected by how the lungs are inflated during fixation. Therefore, we should interpret the results of this study with caution.

The prognostic influence of the number of peritumoral AMs in our study suggests that both intratumoral and the peritumoral microenvironments are important. Recent studies have investigated novel therapeutic strategies aimed at depleting TAMs and/or reprogramming their tumor-promoting effects (Mantovani et al. 2017; Anfray et al. 2020; Sarode et al. 2020). Considering the results of our study, there may be a need for therapeutic efforts targeting AMs, not only TAMs.

\section{Abbreviations}

NSCLC, non-small cell lung cancer; TME, tumor microenvironment; TAM, tumor associated macrophage; AM, alveolar macrophage; AIS, adenocarcinoma in situ; HE, hematoxylin and eosin; VVG, Verhoeff-vanGieson; STAS, spread through air space; TCGA, The Cancer Genome Atlas; GO, Gene Ontology; OS, overall survival; DFS, disease-free survival.

\section{Declarations}

\section{Funding:}

This study was supported in part by the National Cancer Center Research and Development Fund (31-A$6)$.

\section{Conflicts of interest:}

The authors declare that they have no conflict of interest.

\section{Availability of data and material:}

There are no availability of data and material. 
Code availability:

There are no code availability.

\section{Authors' contributions:}

ON and GI participated in the study design, data collection, data analysis, manuscript writing and manuscript review. All other authors participated in manuscript review and revision and provided final approval for submission.

\section{Ethics approval:}

This retrospective study was approved by the Institutional Review Board (IRB approval number; 2020239).

\section{Consent to participate:}

Informed consent was obtained from all patients.

\section{Consent to publication:}

Comprehensive informed consent was obtained from all patients.

\section{References}

1. Siegel R, Miller K, Jamel A (2020) Cancer statistics, 2020. CA caner J Clin 70:7-30.

2. Lewis D, Chen H, Cockburn M, Wu X, Stroup A, et al (2014) Early estimates of SEER cancer incidence, 2014. Cancer 123(13):2524-2534.

3. Rami-Porta R, Bolejack V, Giroux D, Chansky K, Crowley J, et al (2014) The IASLC lung cancer staging project: The new database to inform the eighth edition of the TNM classification of lung caner. $J$ Thorac Onco 9:1618-1624.

4. Comito G, Ippolito L, Chiarugi P, Cirri P (2020) Nutritional exchanges within tumor microenvironment: Impact for cancer aggressiveness. Front Oncol 10:396.

5. Kalluri R (2016) The biology and function of fibroblasts in cancer. Nat Rev Cance 16:582-598.

6. Shinghal S, Stadanlick J, Annunziata M, Rao A, Bhojnagarwala, et al (2019) Human tumorassociated monocytes/macrophages and their regulation of $\mathrm{T}$ cell responses in early-stage lung cancer. Sci Transl Med11(479):eaat1500.

7. Ishii G, Ochiai A, Neri S (2016) Phenotypic and functional heterogeneity of cancer-associated fibroblast within the tumor microenvironment. Advanced Drug Delivery Reviews. Volume 99, Part B Pages 186-196.

8. Hwang L, Kim J, Ylaya K, Chung E, Kitano H, et al (2020) Tumor-associated macrophage, angiogenesis and lymphangiogenesis markers predict prognosis of non-small cell lung cancer 
patients. J Transl Med 18:443.

9. Lewis C, Pollard J (2006) Distinct role of macrophages in different tumor microenvironments. Cancer Res 66:605-612.

10. Bingle L, Brown N, Lewis C (2002) The role of tumour-associated macrophages in tumour progression: implications for new anticancer therapies. J Pathol 196:254-265.

11. Mantovani A, Marchesi F, Maleschi A et al (2017) Tumor-associated macrophages as treatment targets in oncology. Nat Rev Clin Oncol 14(7):399-416.

12. Conway E, Pikor L, Kung S, Hamilton M, Lam S, et al (2016) Macrophages, inflammation, and lung cancer. Am J Respir Crit Care Med 193(2):116-30.

13. Ohtaki Y, Ishii G, Nagai K, Ashimine S. Kuwata T, et al (2010) Stromal Macrophage expressing CD204 is associated with tumor aggressiveness in lung adenocarcinoma. J Thorac Oncol 5:1507-1515.

14. Hirayama S, Ishii G, Nagai K, Ono S, Kojima M, Yamauchi C, et al (2012) Prognostic impact of CD-204 positive macrophages in lung squamous cell carcinoma. J Thorac Oncol 7:1790-1797.

15. Infante J, Matsubayashi H, Sato N, Tnascia J, Klein A, et al (2007) Peritumoral fibroblast SPARC expression and patient outcome with resectable pancreatic adenocarcinoma. J Clin Oncol 25:319325.

16. Auvinen P, Tammi R, Parkkinen J, Tammi M, Agren U, et al (2000) Hyaluronan in peritumoral stroma and malignant cells associates with breast cancer spreading and predicts survival. Am J Pathol 156(2):529-536.

17. Kuang D, Peng C, Zhao Q, Wu Y, Chen M, et al (2010) Activated monocytes in peritumoral stroma of hepatocellular carcinoma promote expansion of memory T helper 17 cells. Hepatology 52(1):154-64.

18. Iriki T, Ohnishi K, Fujiwara Y, Horlad H, Saito Y et al (2017) The cell-cell interaction between tumorassociated macrophages and small cell lung cancer cells is involved in tumor progression via STAT3 activation. Lung cancer 106:22-32.

19. Lee Y, Jeong J, Nyenhuis S, Berdyshev E, Chung S, et al (2015) Recruited alveolar macrophages, in response to airway epithelial-derived monocyte chemoattractant protein 1/CCL2, regulated airway inflammation and remodeling in allergic asthma. Am J Respir Cell Mol Biol 52(6):772-84.

20. Allard B, Panariti A, Martin J (2018) Alveolar macrophages in the resolution of inflammation, tissue repair, and tolerance to infection. Front Oncol 9:1777.

21. Guilliams M, Kleer I, Henri S, Post S, Vanhoutte L, et al (2013) Alveolar macrophages develop from fetal monocytes that differentiate into long-lived cells in the first week of life via GM-CSF. J Exp Med 210 (10):1977-1992.

22. Pollard JW (2004) Tumour-educated macrophages promote tumour progression and metastasis. Nat Rev Cancer 4(1):71-78.

23. Sica A, Porta C, Morlacchi S, Banfi S, Strauss L, et al (2012) Origin and functions of tumo-associated myeloid cells (TAMCs). Cancer Microenviron 5(2):133-49. 
24. Sharma S, Chintala N, Vadrevu S, Patel J, Karbowniczek M, et al (2015) Pulmonary alveolar macrophages contribute to the premetastatic niche by suppressing antitumor $\mathrm{T}$ cell responses in the lungs. J Immunol 194:5529-5538.

25. Nosaka T, Baba T, Tanabe Y, Sasaki S, Nishimura T, et al (2018) Alveolar macrophages drive hepatocellular carcinoma lung metastasis by generating leukotriene B4. J Immunol 200:1839-1852.

26. Speth J, Penke L, Bazzill J, Park K, Rubio R, et al (2019) Alveolar macrophage secretion of vesicular SOCS3 represents a platform for lung cancer therapeutics. JCI Insight 4(20):e131340.

27. Anfray C, Ummarino A, Andon F, Allavena P (2020) Current strategies to target tumor-associatedmacrophages to improve anti-tumor immune responses. Cells 9(1):46.

28. Sarode P, Zheng X, Gitopoulou G, Weigert A, Kuenne C, et al (2020) Reprogramming of tumorassociated macrophages by targeting $\beta$-catenin/FOSL2/ARID5A signaling: A potential treatment of lung cancer. Sci. Adv 6:eaaz6105.

\section{Figures}




\section{Figure 1}
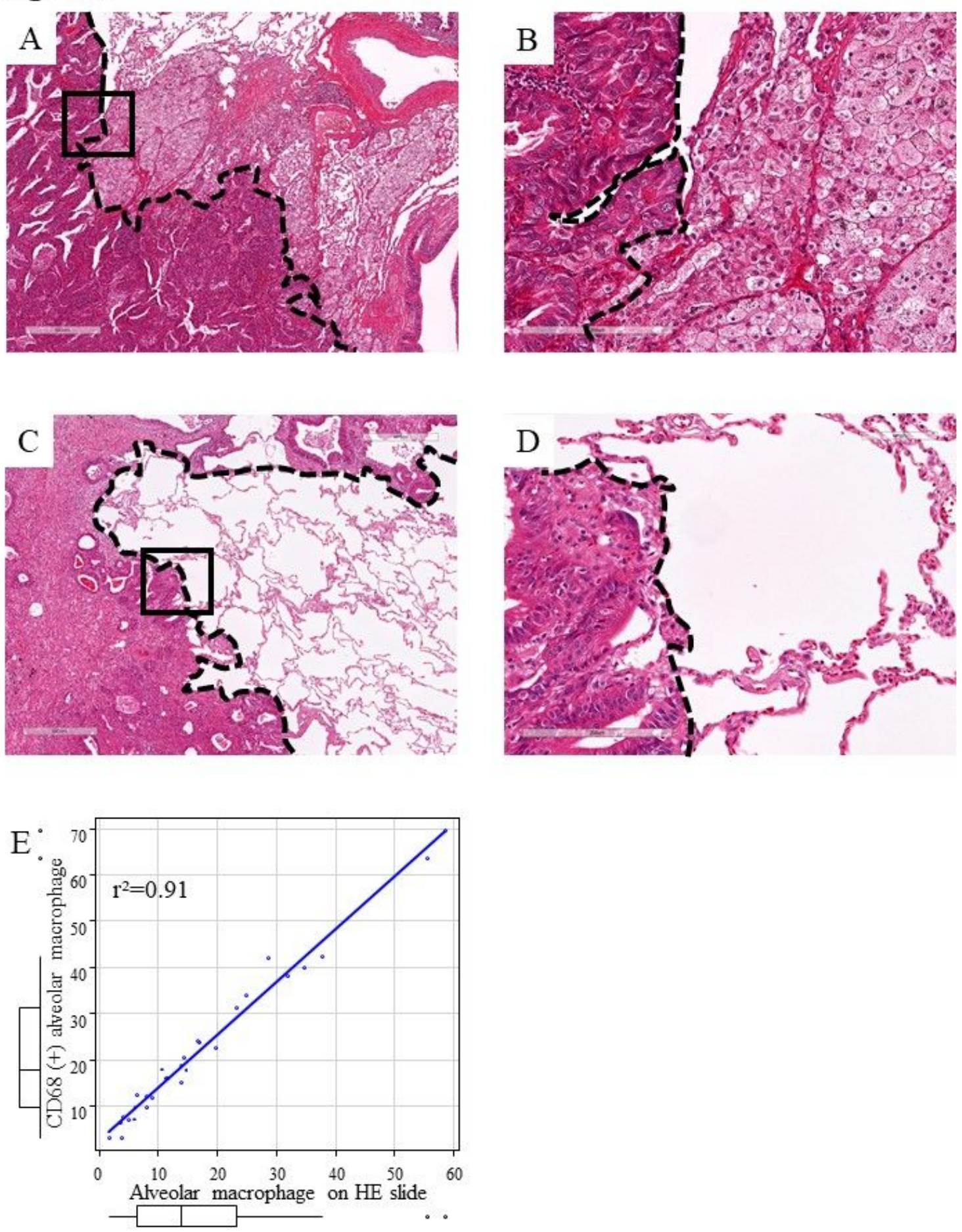

\section{Figure 1}

Microscopic features of high and low peritumoral macrophage contents. (A) Low-power view, and (B) high-power view of a high peritumoral macrophage content. (C) Low-power view, and (D) high-power view of a low peritumoral macrophage content. (E) Correlation between the number of peritumoral alveolar macrophages on HE slides and CD68 immunostaining slides. The x-axis shows the number of 
peritumoral AMs on the HE slide, and the $y$-axis shows the number of peritumoral CD68-positive AMs. AMs, alveolar macrophages; $\mathrm{HE}$, hematoxylin and eosin

\section{Figure 2}

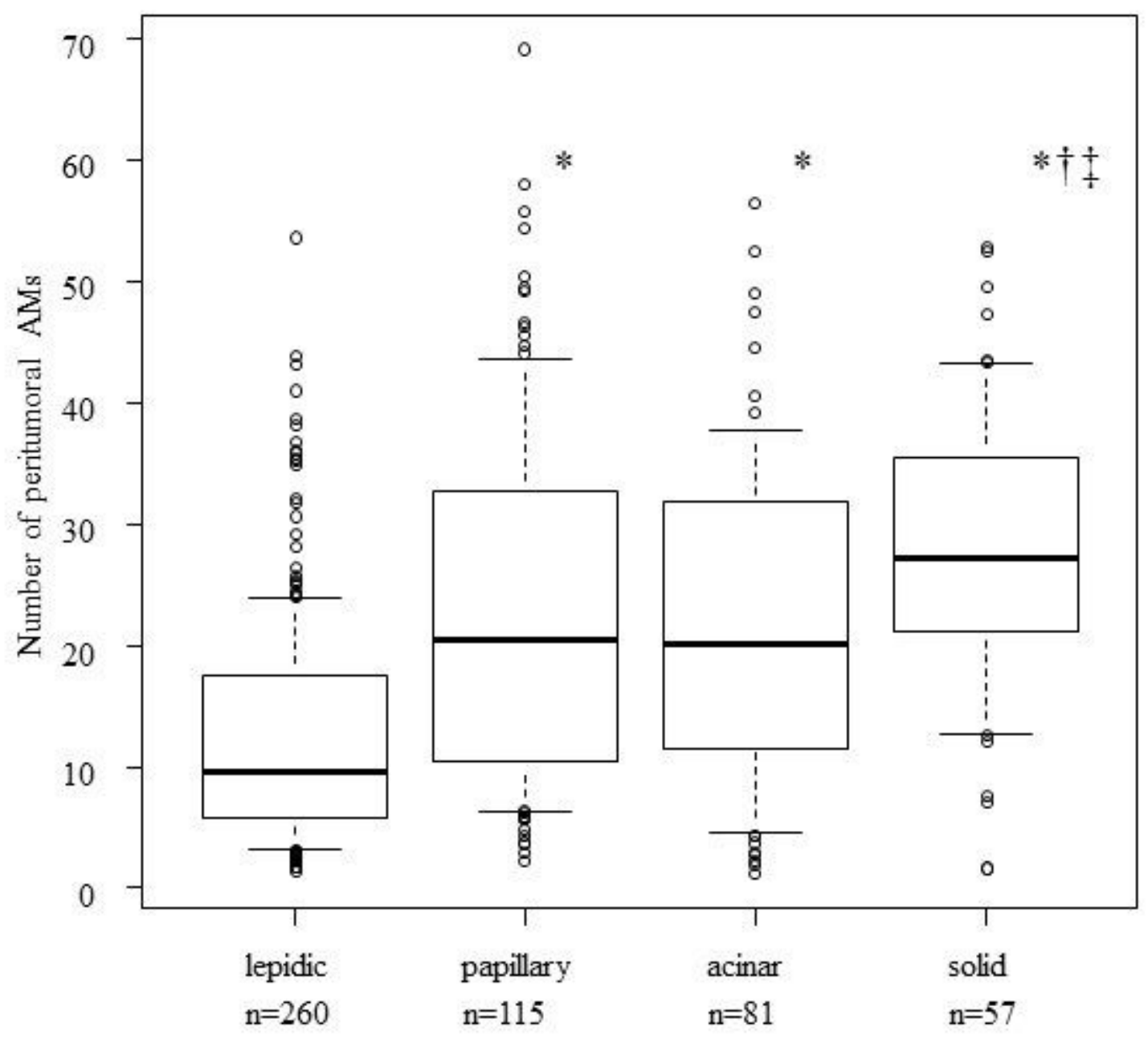

* $p<0.01$ compared with lepidic

$\dagger p<0.01$ compared with papillary

$\ddagger p<0.01$ compared with acinar

\section{Figure 2}

Numbers of peritumoral AMs in each predominant subtypes: lepidic, papillary, acinar, and solid. Statistical analysis was conducted in each two groups. 
Figure 3

A
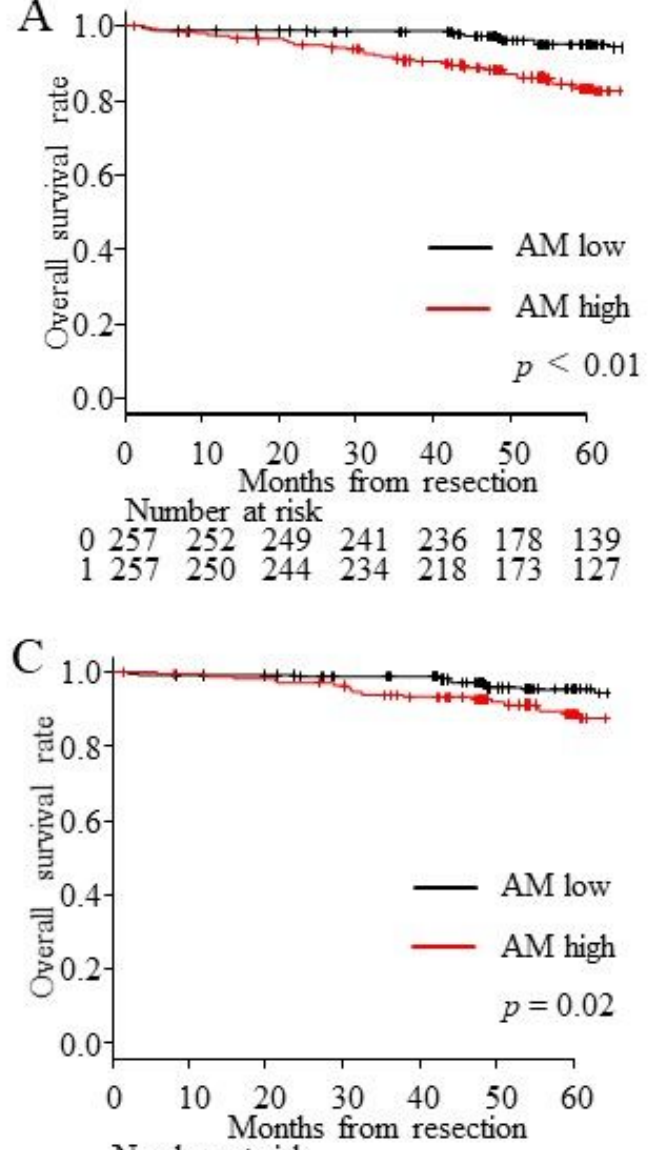

Number at risk

$\begin{array}{llllllll}0 & 226 & 223 & 221 & 213 & 208 & 156 & 123 \\ 1 & 170 & 167 & 165 & 161 & 151 & 121 & 94\end{array}$

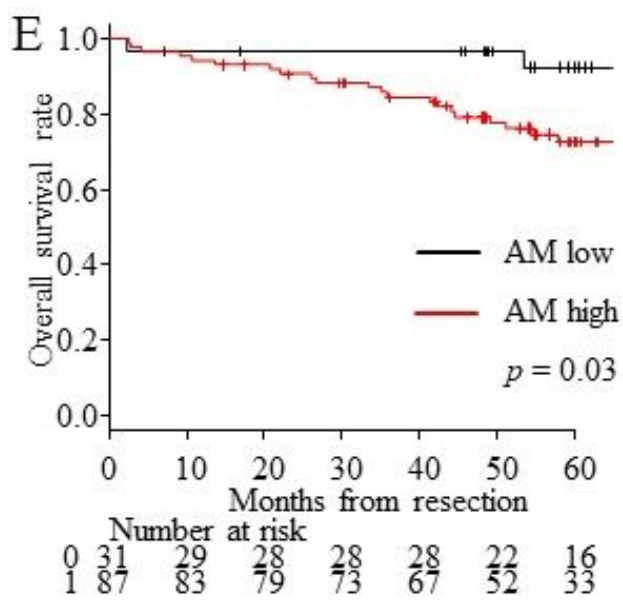

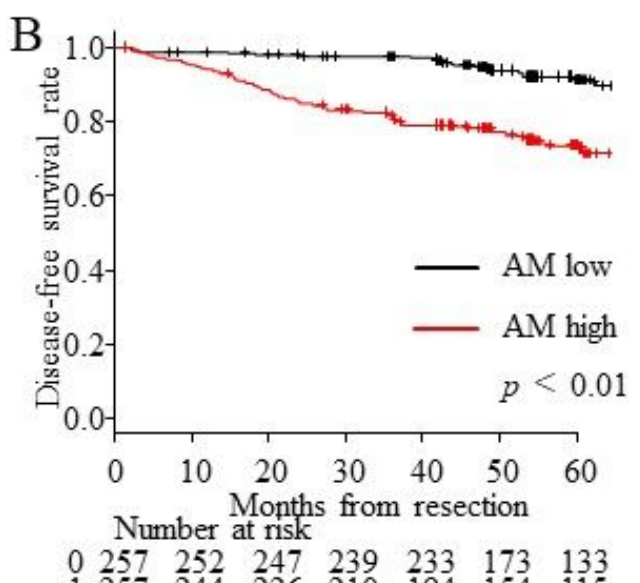

$\begin{array}{llllllll}0 & 257 & 252 & 247 & 239 & 233 & 173 & 133 \\ 1 & 257 & 244 & 226 & 210 & 194 & 154 & 115\end{array}$

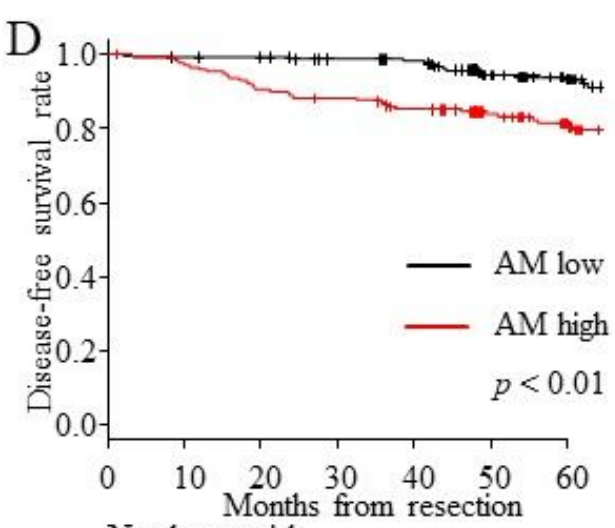

Number at risk

$\begin{array}{llllllll}0 & 226 & 223 & 221 & 213 & 207 & 154 & 120 \\ 1 & 170 & 164 & 153 & 148 & 140 & 113 & 89\end{array}$

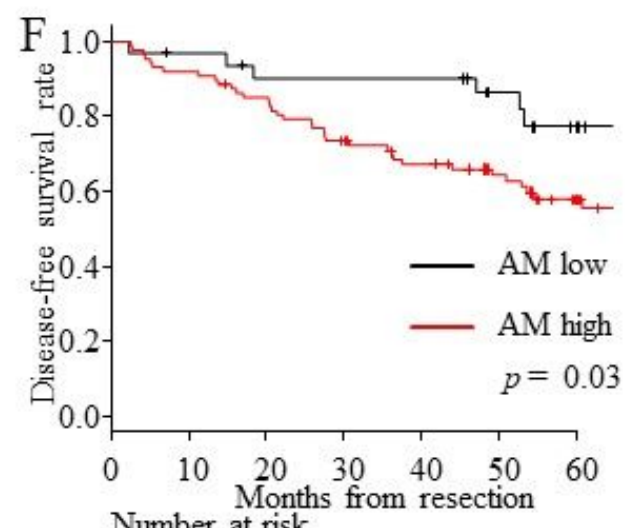

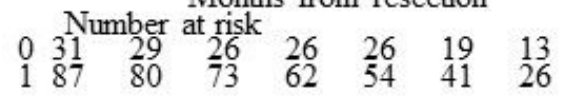

\section{Figure 3}

Kaplan-Meier analysis according to the number of peritumoral AMs. (A) Overall survival (OS), (B) Diseasefree survival (DFS) in stage I patients. (C) OS, (D) DFS in stage IA patients. (E) OS, (F) DFS in stage IB patients. AMs, alveolar macrophages 

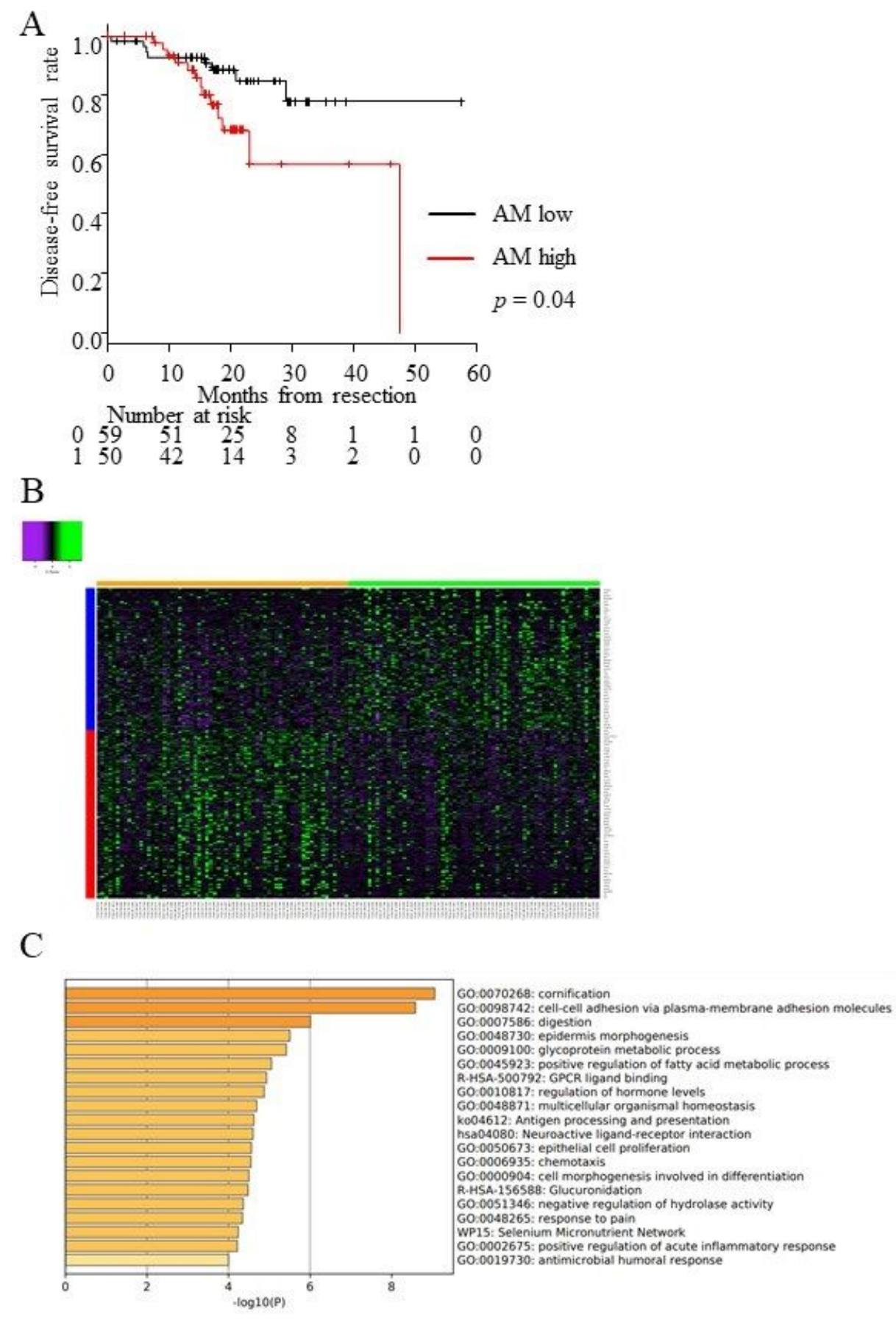

\section{Figure 4}

Survival and gene ontology analyses by the number of peritumoral AMs in the TCGA database. (A)Kaplan-Meier analysis of disease-free survival in the TCGA database. (B)Heat map compared with higher and lower peritumoral AM content. (C)Gene ontology and pathways were significantly higher in high peritumoral AM content than in low peritumoral AM content. 


\section{Supplementary Files}

This is a list of supplementary files associated with this preprint. Click to download.

- AMpaper.SuppFigure1.pptx

- AMpaper.SuppFigure2.pptx

- AMpaper.SuppFigure3.pptx

- Supp.Table1.AMpaper.Noritake.docx

- SuppTable2.AMpaper.Noritake.docx 